\title{
Spinning disk for compressive imaging
}

\author{
H. Shen, ${ }^{1}$ L. Gan, ${ }^{2}$ N. Newman, ${ }^{1}$ Y. Dong, ${ }^{1}$ C. Li, ${ }^{1}$ Y. Huang, ${ }^{1}$ and Y. C. Shen ${ }^{1, *}$ \\ ${ }^{1}$ Department of Electrical Engineering and Electronics, University of Liverpool, Liverpool L69 3GJ, UK \\ ${ }^{2}$ Electronic and Computer Engineering, Brunel University, Uxbridge UB8 3PH, UK \\ ${ }^{*}$ Corresponding author: ycshen@liverpool.ac.uk
}

Received November 2, 2011; accepted November 10, 2011;

posted November 17, 2011 (Doc. ID 157555); published December 22, 2011

\begin{abstract}
We report the first, to the best of our knowledge, experimental implementation of a spinning-disk configuration for high-speed compressive image acquisition. A single rotating mask (i.e., the spinning disk) with random binary patterns was utilized to spatially modulate a collimated terahertz (THz) or IR beam. After propagating through the sample, the THz or IR beam was measured using a single detector, and THz and IR images were subsequently reconstructed using compressive sensing. We demonstrate that a 32-by-32 pixel image could be obtained from 160 to 240 measurements in both the IR and THz ranges. This spinning-disk configuration allows the use of an electric motor to rotate the spinning disk, thus enabling the experiment to be performed automatically and continuously. This, together with its compact design and computational efficiency, makes it promising for real-time imaging applications. (c) 2011 Optical Society of America

OCIS codes: $110.6795,320.7100$.
\end{abstract}

Over the past decade, there has been great interest in the study of compressive imaging [ $\underline{1}-\underline{6}]$, where the number of physical measurements is much smaller than that of pixels in the reconstructed image. Such systems may find applications for imaging at wavelengths that are currently impossible with conventional CCD and complementary metal-oxide semiconductor imagers. For instance, at spectral ranges of IR, sampling a continuous object can be very expensive where exotic detector materials are used $[1,2]$. As another example, at terahertz (THz) range, conventional $\mathrm{THz}$ time-domain imaging systems remain too slow for time-critical applications due to their pixel-by-pixel raster-scanning nature [주느]

Consider an image with $N$ pixels in total and let $x$ represent its vector version. The compressive linear imaging process can be described as [1]

$$
y_{M \times 1}=\Phi_{M \times N} x_{N \times 1}+n_{M \times 1}
$$

where $y$ denotes the measured signal with length of $M$ $(M \ll N), \Phi$ corresponds to the $M \times N$ sampling operator, and $n$ represents the measurement noise. One fundamental question in the research of compressive imaging is the design and implementation of the sampling operator $\Phi$. Recently, the discovery of compressive sensing (CS) theory $[\underline{7}, \underline{8}]$ has triggered the study of random projection for compressive imaging. $\mathrm{CS}$ is based on the sparse signal model, in which a length- $N$ signal can be represented or well approximated with only $K$ coefficients in a certain transform domain. It was shown in $[\underline{7}, 8]$ that, when $\Phi$ is a fully random Gaussian or Bernoulli operator, a sparse signal $x$ can be faithfully reconstructed from $y$ if $M$ satisfies $M \geq O(K \log (N / K))$.

Motivated by the CS theory, several single-pixel cameras [2-6] have been developed. Most of existing works use a random Bernoulli operator, in which the entries of $\Phi$ are randomly selected as 0 and 1 with equal probability. In such systems, a set of $M$ independent twodimensional random binary masks are required, each of which corresponds to one row of $\Phi$. Despite their theoretical advantages, there are a couple of practical limitations. First, as each row of $\Phi$ is independent, the imaging speed is limited by the slow translation of one random pattern to another [ $\underline{3}, \underline{4}]$. Second, a fully random binary operator incurs high computational complexity and huge memory, especially for high-resolution imaging [5] .

In a previous work [6], we proposed the use of a single rotating disk (similar to the Nipkow disk used in confocal microscopy [9]) for a fast single-pixel camera. The measurements in [6] were performed in a "stopmeasure-rotate" fashion by using a motorized stage to rotate the disk, and terahertz $(\mathrm{THz})$ images were obtained using a backward wave oscillator source operating at a single frequency of $0.14 \mathrm{THz}$. In this Letter, we show for the first time, to our knowledge, that a continuously spinning disk can be used for fast compressive IR and THz time-domain (pulsed) imaging.

The schematic diagram of a spinning-disk configuration is shown in Fig. 1. This disk has random binary patterns, where 0 (the black pixel) and 1 (the white pixel) have equal probabilities. During the imaging process, the whole disk is covered except for a small fixed rectangular area. Each time the disk is rotated by $\alpha$ deg, the inner product between the object and the rectangular area is measured using a single detector. As a further illustration, we provide a video demonstration (Media 1) to explain the spinning-disk imaging process, and to demonstrate how the quality of the reconstructed images progressively improves as the number of measurements increased. Note that, at each rotation angle, the new binary pattern inside the rectangular window is a shifted version of the previous pattern with only a small number of elements added in. The corresponding sampling operator $\Phi$ could thus be approximated as a Toeplitz block matrix. Several groups have studied the feasibility of using Toeplitz and circulant operators for compressive imaging [10-12]. Theoretical [12] and simulation results [ $[\underline{5}, \underline{13}]$ revealed that they can offer similar performance as those of fully random operators at much reduced implementation cost.

In this Letter, we aim to demonstrate experimentally the promising application of a continuously spinning disk for compressive IR and THz time-domain (pulsed) imaging. The general experimental setup is shown in Fig. 1 . 


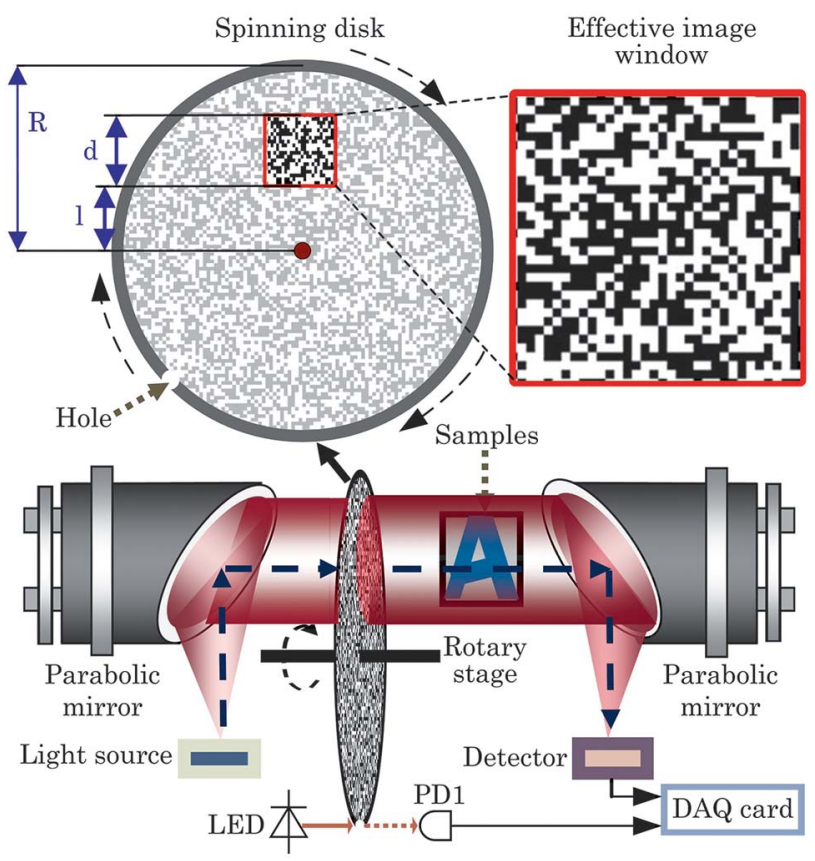

Fig. 1. (Color online) Schematic diagram of a spinningdisk-based compressive imaging system. The square enclosed by the solid red line represents the "effective image window." In our experiment, the spinning disk has a radius of $R=95 \mathrm{~mm}$ and the image window is placed $l=35 \mathrm{~mm}$ from the center of the disk. A hole of $0.5 \mathrm{~mm}$ diameter at the edge of the spinning disk is used for synchronizing the mask position and the measured signal. LED, light emitting diode; PD1, photodiode; DAQ, data acquisition (Media 2).

The IR light (or THz wave) from a source was collimated using a parabolic mirror. After propagating through the sample and the spinning disk, the IR (or THz) beam was then focused, by using another parabolic mirror, onto the detector.

In our experiments, all the samples were made of copper tape on a plastic plate. The spinning disk has a radius of $R=95 \mathrm{~mm}$ and the imaging window is placed $l=$ $35 \mathrm{~mm}$ from the center of the disk (Fig. 1). The effective image window has a dimension of $32 \mathrm{~mm} \times 32 \mathrm{~mm}$, with each pixel of size $1 \mathrm{~mm} \times 1 \mathrm{~mm}$. Hence, the reconstructed image is of size $32 \times 32$ (1024 pixels in total). The spatial resolution of the image is mainly limited by the pixel size, which is much larger than the wavelength of the IR and $\mathrm{THz}$ radiation used here.

One important issue is how to synchronize the disk rotation with data measurement. To achieve this, we put a hole of $0.5 \mathrm{~mm}$ diameter near the edge of the spinning disk. An LED and a photodiode (PD1 in Fig. 1) were then used to monitor the disk rotation. The signal recorded using PD1 provides information about the relative mask positions for the data acquisition (DAQ) card. Hence, the system can effectively measure the CS samples while continuously spinning the disk.

Two reconstruction algorithms, i.e., the minimum mean square error (MMSE) linear estimation $[1,14]$ and the total variation minimization (TV-min) nonlinear reconstruction algorithm [8] have been investigated. Note that CS requires nonlinear reconstruction [묘, $\underline{]}$. However, algorithms like TV-min optimization are time consuming. To keep up with the measurement speed, we use the classical MMSE linear operator [1,14] as an initial solution, in which the autocorrelation matrix of $x$ follows the autoregressive model of order 1 with the autocorrelation coefficient $\rho=0.95$ [14]. The time required to reconstruct a $32 \times 32$ pixel image using MMSE reconstruction is only about $0.002 \mathrm{~s}$, while that using TV-min optimization is around $5 \mathrm{~s}$ (hardware specifications: Intel Core2 Duo CPU at $2.00 \mathrm{GHz}, 3.00$ Gbyte memory; software: MATLAB 2011a).

In IR compressive imaging experiments, an IR LED and a photodiode were used as the light source and the detector, respectively. The spinning disk was fabricated by simply printing the designed binary patterns on a transparent plastic sheet. Figure 2 shows, as examples, the IR images of English characters T, E, R, and A, and a Chinese character guo reconstructed from 240 measurements. One can see that, even with MMSE linear reconstruction, all the characters (including the complicated guo) are visually recognizable. As expected, better quality images could be obtained using the TV-min optimization algorithm. The imaging acquisition speed is about 1 image/s, mainly limited by the speed of the specific electric motor (940D series, MFA) used in our experiment.

In $\mathrm{THz}$ compressive imaging experiments, we used a typical $\mathrm{THz}$ time-domain imaging system [4]. The $\mathrm{THz}$ emitter was a biased photoconductive antenna [15] and the $\mathrm{THz}$ receiver was a $\mathrm{ZnTe}$ electro-optic crystal [16]; both of them were driven by femtosecond laser pulses from a Ti:sapphire laser. In the experiments, we first found the maximum $\mathrm{THz}$ signal (peak position) by varying the time delay between the THz pulse and the optical probe pulse using a variable delay stage. We then fix the position of the variable delay stage at the $\mathrm{THz}$ peak position, and record the THz signal as a function of the rotation angle of the spinning disk, which, in this case, was driven by a motorized rotation stage (CR1-Z7 series, Thorlabs). The random binary pattern of the spinning disk was fabricated on a standard print circuit board (PCB). We found that the PCB substrate material absorbs strongly $\mathrm{THz}$ radiation at frequencies above $1 \mathrm{THz}$. Consequently, the $\mathrm{THz}$ signal reduced significantly after transmitting through the spinning disk, and the useful frequency range was below 0.8 THz. Owing to the limited

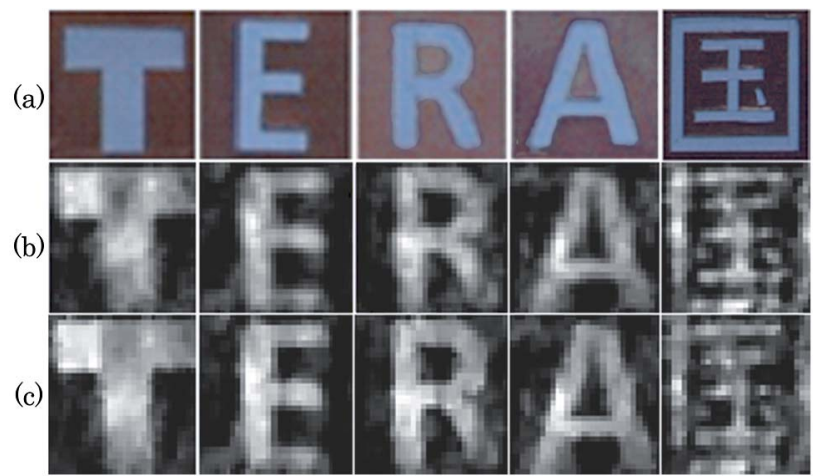

Fig. 2. (Color online) (a) Photographs of the samples used in IR imaging experiment, and their corresponding IR images reconstructed using (b) MMSE linear estimation and (c) the TV-min nonlinear reconstruction algorithm. Each image has $32 \times 32$ pixels and was reconstructed from 240 measurements. 
(a)

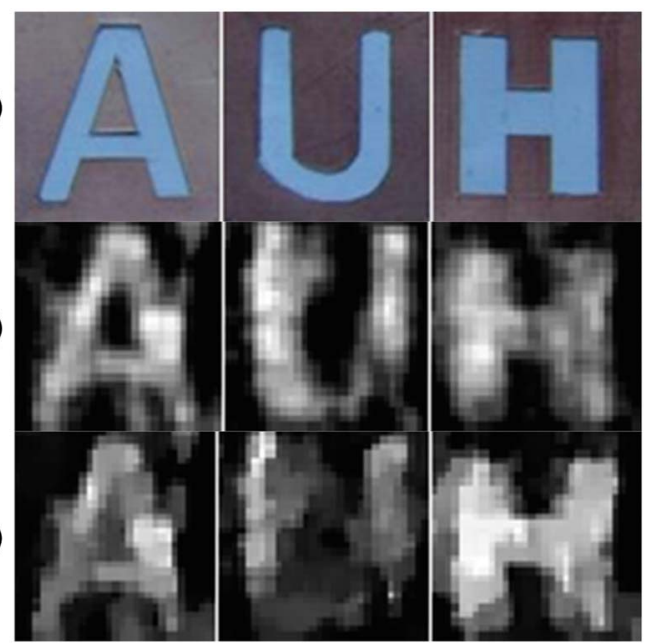

Fig. 3. (Color online) (a) Photographs of the samples used in the $\mathrm{THz}$ imaging experiment, and their corresponding $\mathrm{THz}$ images reconstructed using (b) MMSE linear estimation and (c) the TV-min nonlinear reconstruction algorithm. Each image has $32 \times 32$ pixels and was reconstructed from 160 measurements.

signal-to-noise ratio, the rotation speed of the spinning disk was $5 \mathrm{deg} / \mathrm{s}$ and it took about $80 \mathrm{~s}$ to measure one THz image. For the same reason, we used 160 measurements for image reconstruction, as further increase in the measurement number decreases the quality of the reconstructed THz images. In fact, it was also reported in [3] that, even with full random binary operators, more measurements could add more artifacts for reconstructed $\mathrm{THz}$ images. Figure 3 shows our experimental results for THz images of $\mathrm{A}, \mathrm{U}$, and $\mathrm{H}$. Although the quality of reconstructed images is not as good as that in the IR experiments, all these characters are still recognizable. Also, compared with classical MMSE reconstruction, the TV-min optimization does not provide much visual improvement. This could be caused by the imperfection of the operator $\Phi$ in practical implementations. Fabrication error and misalignment of the spinning-disk patterns as well as the nonuniform disk substrate could all contribute to such noises in $\Phi$. Nevertheless, our experimental results demonstrated the concept of using a continuously spinning-disk approach for rapid compressive $\mathrm{THz}$ imaging.

Recall that, in $[3,4]$, a set of $10 \mathrm{~s}$ or $100 \mathrm{~s}$ of physical masks were used and it could take up to $30 \mathrm{~min}$ [4] to complete the $\mathrm{THz}$ image acquisition by switching 40 masks manually. Our spinning-disk approach uses only one physical mask. This simplifies the experimental setup, and allows the measurements to be done continuously. We noted that a $\mathrm{THz}$ spatial modulator could, in principle, be used for automatic CS-THz imaging [17]. However, the $\mathrm{THz}$ spatial modulator reported has only $4 \times 4$ pixels, limiting its practical imaging applications.

In conclusion, we have reported the design and implementation of a continuously spinning-disk-based compressive imaging system. Such an approach offers the advantages of compact design, fast computation, and easy implementation with potential video-rate imaging speed. As demonstrated here, the idea of the spinning-disk approach is applicable to both IR and $\mathrm{THz}$ time-domain imaging. In particular, for a $32 \times 32 \mathrm{im}$ age, 240 measurements (i.e., around $24 \%$ sampling rate) are sufficient to get an IR image with reasonably good quality. For THz imaging, 160 measurements (about $16 \%)$ can provide images with acceptable quality. The $\mathrm{THz}$ image quality and acquisition speed could be significantly improved by using a spinning disk with a substrate material more transparent to $\mathrm{THz}$ radiations.

This work is partially supported by the Engineering and Physical Sciences Research Council (EPSRC, EP/ $\mathrm{H} 02235 \mathrm{X} / 1$ and EP/I038853/1), and the EPSRC laser loan pool scheme. N. Newman would like to acknowledge funding from the EPSRC Vacation Bursary Scheme.

\section{References}

1. J. Ke and M. A. Neifeld, Appl. Opt. 46, 5293 (2007).

2. M. F. Duarte, M. A. Davenport, D. Takhar, J. N. Laska, T. Sun, K. F. Kelly, and R. G. Baraniuk, IEEE Signal Process. Mag. 25(2), 83 (2008).

3. W. L. Chan, K. Charan, D. Takhar, K. F. Kelly, R. G. Baraniuk, and D. M. Mittleman, Appl. Phys. Lett. 93, 121105 (2008).

4. Y. C. Shen, L. Gan, M. Stringer, A. Burnett, K. Tych, H. Shen, J. E. Cunningham, E. P. J. Parrott, J. A. Zeitler, L. F. Gladden, E. H. Linfield, and A. G. Davies, Appl. Phys. Lett. 95, 231112 (2009).

5. A. Heidari and D. Saeedkia, in 34th International Conference on Infrared, Millimeter, and Terahertz Waves (IEEE, 2009), Vol. 1-2, pp. 694-695.

6. H. Shen, N. Newman, L. Gan, S. C. Zhong, Y. Huang, and Y. C. Shen, in 35th Intermational Conference on Infrared, Millimeter, and Terahertz Waves (IEEE, 2010), pp. 1-2.

7. D. Donoho, IEEE Trans. Inf. Theory 52, 1289 (2006).

8. E. Candes, J. Romberg, and T. Tao, IEEE Trans. Inf. Theory 52, 489 (2006).

9. G. Q. Xiao, T. R. Corle, and G. S. Kino, Appl. Phys. Lett. 53, 716 (1988).

10. J. Haupt, W. U. Bajwa, G. Raz, and R. Nowak, IEEE Trans. Inf. Theory 56, 5862 (2010).

11. F. Sebert, Y. M. Zou, and L. Ying, in Proceedings of International Conference on Information Technology and Application and Biomedicine (IEEE, 2008), pp. 47-50.

12. H. Rauhut, in Theoretical Foundations and Numerical Methods for Sparse Recovery, Vol. 9 of Radon Series for Computational and Applied Mathematics, M. Fornasier, ed. (de Gruyter, 2010), pp. 1-92.

13. W. Yin, S. P. Morgan, J. Yang, and Y. Zhang, Rice University CAAM Technical Report TR10-01 (2010).

14. L. Gan, in 15th International Conference on Digital Signal Processing (IEEE, 2007), pp. 403-406.

15. D. H. Auston, K. P. Cheung, and P. R. Smith, Appl. Phys. Lett. 45, 284 (1984).

16. Q. Wu and X. C. Zhang, Appl. Phys. Lett. 71, 1285 (1997).

17. W. L. Chan, H. T. Chen, A. J. Tayler, I. Brener, M. J. Cich, and D. M. Mittleman, Appl. Phys. Lett 94, 213511 (2009). 